Kompass

Neumología

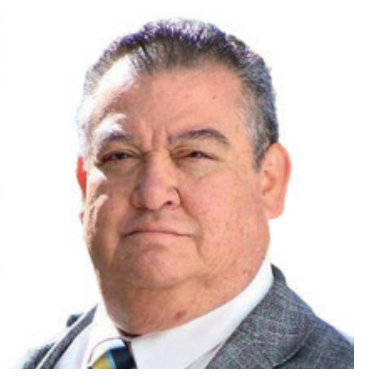

\title{
Sueño y ritmos circadianos en supervivientes de falla respiratoria aguda
}

\author{
Jesús José Arzola-Rodríguez
}

Neurólogo, Egresado Centro Médico Nacional La Raza IMSS, Académico de la Facultad de Medicina y Nutrición de la Universidad Juárez del Estado de Durango, Durango, México

Resumen de Yang PL, Ward TM, Burr RL, et al.: Sleep and Circadian Rhythms in Survivors of Acute Respiratory Failure. Front Neurol. 2020; 11:94 (DOI: 10.3389/fneur.2020.00094).

\section{Keywords}

Anti-neutrophil cytoplasmic autoantibody · Elderly .

Immunosuppression · Outcomes · Vasculitis

\section{Abstract}

Background: Little is known about sleep and circadian rhythms in survivors of acute respiratory failure (ARF) after hospital discharge.

Objectives: To examine sleep and rest-activity circadian rhythms in ARF survivors 3 months after hospital discharge, and to compare them with a community-dwelling population.

Methods: Sleep diary, actigraphy data, and insomnia symptoms were collected in a pilot study of 14 ARF survivors. Rest-activity circadian rhythms were assessed with wrist actigraphy and sleep diary for 9 days, and were analyzed by cosinor and non-parametric circadian rhythm analysis.

Results: All participants had remarkable actigraphic sleep fragmentation, $71.5 \%$ had subclinical or clinical insomnia symptoms. Compared to community-dwelling adults, this cohort had less stable rest-activity circadian rhythms $(p<0.001)$, and weaker circadian strength $(p<0.001)$.

Conclusion: Insomnia and circadian disruption were common in ARF survivors. Sleep improvement and circadian rhythm regularity may be a promising approach to improve quality of life and daytime function after ARF.

(c) 2020 Yang, Ward, Burr, Kapur, McCurry, Vitiello, Hough and Parsons. 


\section{Transferencia en la práctica}

\section{Contexto del estudio}

Los conocimientos que tenemos sobre el sueño y los ritmos circadianos en supervivientes de insuficiencia respiratoria aguda (IRA) tras el alta hospitalaria, son escasos. Examinar los ritmos circadianos del sueño-vigilia y la actividad en reposo en supervivientes de IRA, 3 meses después del alta hospitalaria y compararlos con una población que vive en la comunidad, fue el objetivo del estudio de Pei-Lin Yang y colegas comentado aquí. Se recogió el diario del sueño, donde el paciente registra los mayores detalles posibles, como la hora en que va a la cama, cuánto tarda en conciliar el sueño, si es o no fragmentado, a qué horas despierta y se siente con la suficiente energía durante las primeras horas o durante todo el día y algunas observaciones particulares del paciente, los datos de actigrafía (dispositivo tipo reloj de pulsera, que registra los movimientos de la extremidad y del cuerpo, día y noche, por un periodo de tiempo determinado) y los síntomas del insomnio. En el presente estudio piloto de 14 supervivientes de IRA, los ritmos circadianos se evaluaron con los métodos mencionados, durante 9 días y se analizaron mediante el modelo matemático de ritmo circadiano COSINOR (modelo matemático que permite la representación de la oscilación cronobiológica de una variable con comportamiento rítmico).

\section{Resultados del Estudio}

Todos los participantes tenían una notable fragmentación actigráfica del sueño, el 71,5\% tenía síntomas de insomnio subclínico o clínico. En comparación con los adultos que viven en la comunidad, esta cohorte tenía ritmos circadianos de actividad en reposo menos estables $(p<0,001)$ y una fuerza circadiana más débil $(p<0,001)$. El estudio, aunque con un grupo de 14 pacientes, (no es tarea fácil reunir un grupo grande de pacientes sobrevivientes de un IRA), concluye en forma bastante objetiva, basada en variables, algunas un poco subjetivas, como las consignadas en el libro del sueño y los síntomas de insomnio y otras más objetivas, tangibles, como la actividad física registrada en actigráfica y analizado por el método COSINOR, que los pacientes sobrevivientes de IRA, a noventa días de su egreso hospitalario, presentaban alteraciones del ritmo circadiano y del sueño, con fragmentación del mismo y signos clínicos o sub clínicos de Insomnio, comparados con adultos que habitan la misma comunidad.

\section{Conclusiones y recomendaciones para la práctica}

El presente estudio es bastante sencillo y no requiere de equipos sofisticados, de alta tecnología y costos muy elevados, sin embargo sus resultados son muy interesantes, ya que desde hace tiempo, suponíamos que los pacientes que sufrieron episodios importantes y que son sobrevivientes de Insuficiencia Respiratoria Aguda, tendrían repercusiones sobre el ritmo circadiano, especialmente en los ciclos sueño-vigilia de actividad en reposo. Este estudio, nos permite documentar en forma objetiva, ordenada y tangible, el comportamiento de esas variables, a 90 días posteriores a su egreso hospitalario, lo cual parece un plazo razonable para haber superado la crisis y aceptado lo ocurrido.

El estudio tiene utilidad, porque sus resultados nos ayudan a tomar medidas que mejoren la calidad de sueño de estos pacientes y elevar su calidad de vida, pertinente porque en cualquier localidad se pueden presentar casos de IRA y es muy importante conocer y manejar las secuelas de los supervivientes, practico, porque implementarlo es relativamente sencillo, no invasivo y se realiza en el hogar de los participantes, pero, sobre todo factible de realizar en un medio en el que los recursos, económicos, tecnológicos, humanos y de infraestructura, son limitados, como pudiera ocurrir en muchas comunidades latinoamericanas.

\section{Disclosure Statement}

Por medio de la presente, el autor declara, no tener ningún conflicto de interés en la presente transferencia de conocimiento.

\section{Referencias}

1 Dodoo-Schittko F, Brandstetter S, Blecha S, et al.: Determinants of quality of life and return to work following acute respiratory distress syndrome: a systematic review. Dtsch Arztebl Int. 2017;114:103-109.

2 Rubenfeld GD, Herridge MS: Epidemiology and outcomes of acute lung injury. Chest. 2007;131:554-562.

3 Herridge MS, Moss M, Hough CL, et al.: Recovery and outcomes after the acute respiratory distress syndrome (ARDS) in patients and their family caregivers. Intensive Care Med. 2016;42:725-738.

4 Bein T, Weber-Carstens S, Apfelbacher C: Long-term outcome after the acute respiratory distress syndrome: different from general critical illness? Curr Opin Crit Care. 2018;24:35-40.

5 Rawal G, Yadav S, Kumar R: Post-intensive care syndrome: an overview. J Transl Int Med. 2017;5:90-92.

Correspondencia: Dr. Jesús José Arzola-Rodríguez, Calle Toronja 307, Fraccionamiento La Glorieta, 34207 Durango, Dgo., México, jjarzolaneuro@yahoo.com.mx 